\title{
Retrieving Memories via Internal Context Requires the Hippocampus
}

\author{
Pamela J. Kennedy and Matthew L. Shapiro \\ Fishberg Research Center for Neurobiology, Kastor Neurobiology of Aging Laboratories, Mount Sinai School of Medicine, New York, New York 10029-6574
}

Episodic memory encodes the unique contexts of events so that people can remember the details of an experience when cued by only a subset of event features (Tulving, 1972). In humans, the hippocampus is crucial for this kind of memory (Scoville and Milner, 1957; Vargha-Khadem et al., 1997). The present study tested whether the hippocampus was required for nonspatial, context-dependent memory retrieval in rats that were trained in a constant external environment to approach different nonspatial goal objects depending on their current internal motivational state (hunger or thirst). The rats learned to reliably approach the correct goal and thus used internal context to guide associative memory retrieval. Both fornix transection and selective neurotoxic hippocampal lesions severely impaired memory performance, but cue and motivational discrimination, as well as stimulus-reward associations, were preserved. The findings suggest that the hippocampus is required for using internal contextual information for flexible associative memory retrieval.

Key words: hippocampus; context; rat; memory; retrieval; lesion

\section{Introduction}

The hippocampus may be crucial for human episodic memory because it supports the rapid acquisition, storage, and retrieval of representations that encode the relationships among items, events, and actions (Eichenbaum, 1999). In animals, debate remains as to whether the hippocampus is specifically involved in the processing and retrieval of spatial information or whether it serves a more general function in learning and memory (O'Keefe and Nadel, 1978; Cohen and Eichenbaum, 1993).

Hirsh (1974) proposed that the hippocampal system is necessary for memory retrieval when it is prompted by contextual cues that refer to, but do not represent explicitly, the items to be remembered. "Contextual memory retrieval" thereby requires conditional operations in which the meaning of a stimulus varies depending on other elements of the situation. Like episodic memory, context-dependent retrieval entails the selective and flexible recovery of relevant information from a collection of common items that may have been encoded during different episodes in the same place. For example, normal rats can learn to use interoceptive states (e.g., hunger or thirst) as contextual cues to direct different goal-oriented spatial responses or to predict shock within ambiguous external environments. Rats with damage to the hippocampal system are impaired at learning these tasks (Hsiao and Isaacson, 1971; Hirsh et al., 1978a,b; Davidson and Jarrard, 1993; Hock and Bunsey, 1998). However, these pre-

Received April 13, 2004; revised June 21, 2004; accepted June 22, 2004.

This work was supported by the National Institutes of Health and the Mount Sinai School of Medicine. We thank Janina Ferbinteanu and Bonnie Fletcher for technical advice, readers for helpful comments on this manuscript, Benjamin Schneider for technical assistance, and Anna Balk for computer programming.

Correspondence should be addressed to Matthew L. Shapiro, Mount Sinai School of Medicine, Neurobiology of Aging Laboratories, 1 Gustave Levy Place EB L9-23, Box 1639, New York, NY 10029-6574. E-mail: matthew.shapiro@mssm.edu.

DOI:10.1523/JNEUROSCI.1388-04.2004

Copyright $\odot 2004$ Society for Neuroscience $\quad 0270-6474 / 04 / 246979-07 \$ 15.00 / 0$ vious acquisition studies did not distinguish learning deficits from impaired contextual retrieval. Moreover, the results were typically interpreted as examples of a more selective deficit, such as in spatial memory (O'Keefe and Nadel, 1978), internal state discrimination (Davidson and Jarrard, 1993), or learning associations between internal and external stimuli (Hock and Bunsey, 1998).

To test whether nonspatial, context-dependent memory retrieval required the hippocampus, rats deprived of food or water on alternate days were trained to enter one of three visually distinct goal boxes to obtain reward. Two goal boxes were consistently paired with either food or water, and a third box was never rewarded. To prevent rats from adopting a spatial response strategy, the goal boxes were moved pseudorandomly among three possible locations at the start of each trial. In this manner, rats presented with identical external cues, including available visible, auditory, and olfactory stimuli, learned to differentially approach and avoid equally reinforced goal objects depending on their current internal context. After the animals maintained a criterion level of performance, they were given complete lesions of either the fornix $(\mathrm{Fx})$ or the hippocampus. Contextual retrieval performance declined to near chance levels, but rats continued to avoid the unrewarded box and showed normal deprivation-based reward preference. The results demonstrate that the hippocampus is necessary for internally cued contextual retrieval of nonspatial memory.

\section{Materials and Methods}

Subjects

Fourteen male Long-Evans rats (Charles River Laboratories, Wilmington, MA) weighing between 250 and $300 \mathrm{gm}$ at the start of training, were housed individually and maintained on a $12 \mathrm{hr}$ light/dark cycle. Training and testing occurred during the light phase. All experiments and surgical procedures were conducted according to guidelines set forth by the In- 
stitutional Animal Care and Use Committee and the National Institutes of Health.

\section{Apparatus}

A wooden radial maze (arms, $54 \mathrm{~cm}$ long, $9 \mathrm{~cm}$ wide; edges, $1 \mathrm{~cm}$ high; elevated $62 \mathrm{~cm}$ above the floor; painted gray) was arranged so that three goal arms separated by $45^{\circ}$ angles were located directly across an octagonal central platform ( $42 \mathrm{~cm}$ across) from a start arm (see Fig. 2). Clear Plexiglas walls $(30 \mathrm{~cm}$ high) with guillotine doors operated by a pulley system surrounded the central platform. Interchangeable goal boxes (30 $\mathrm{cm}$ high, $15 \mathrm{~cm}$ wide, and $13.5 \mathrm{~cm}$ deep), distinguished by color and illumination, were mounted at the ends of each of the goal arms. Within each goal box was a reward well $(0.5 \mathrm{~cm}$ deep and $1.5 \mathrm{~cm}$ in diameter; 4 $\mathrm{cm}$ from the back wall). The apparatus was located in the center of a dark room with black curtains surrounding two-thirds of the maze to minimize access to distal visual cues. A light source mounted above the maze provided dim illumination of the entire apparatus. Small dishes of food and water were placed under each goal box to mask reward-related odor cues.

\section{Training procedure}

Habituation, training, and testing were performed $6 \mathrm{~d}$ per week. During this time, rats were deprived of food and water on alternate days. During habituation, training, and testing, the rats had ad libitum access to the nondeprived item in their home cages. One hour after each daily session, rats were given ad libitum access to both food and water for $2 \mathrm{hr}$ before the removal of the next deprived item. On this regimen, rats maintained at least $80 \%$ of their free-feeding body weight. For each rat, one goal box was paired with $0.2 \mathrm{gm}$ of powdered rat chow, another with four drops of water, and a third had no reward. The specific box-reward pairings were consistent for each rat and counterbalanced among rats. To prevent rats from adopting egocentric or allocentric spatial response strategies, goal boxes were switched among the three goal arms at the start of each trial in a pseudorandom counterbalanced sequence. In this manner, each goal box occupied each arm with the same frequency and sometimes repeated locations from one trial to the next.

Habituation. Rats were handled for $10 \mathrm{~min}$ per day for $4 \mathrm{~d}$ before $4 \mathrm{~d}$ of habituation to the maze. On each day of habituation ( $2 \mathrm{~d}$ per deprivation state), only the goal box designated to hold the reward appropriate to the animal's current deprivation state was baited. Rats were placed on the start arm and allowed to explore the maze until the reward was consumed. Goal boxes were placed in a different arm each day.

Pretraining. Each rat was trained initially to discriminate between the nonrewarded box and the two rewarded goal boxes using a correction procedure. At the start of each trial, a rat was placed on the start arm and two guillotine doors were raised to give access to only two goal arms, one holding the nonrewarded box and one holding the goal box with the reward appropriate to the rat's current deprivation state. A choice was defined when the rat's four paws entered a goal arm. On correct choice trials, the rat was allowed to consume the reward and was then returned to its home cage. The maze was again baited, and the goal boxes were shuffled in preparation for the next trial. On incorrect choice trials, the rat was kept in the nonrewarded box for 5-10 sec and then returned to its home cage. A correction procedure was used after incorrect trials so that the same trial was repeated and the boxes were not shuffled until the rat made the correct choice. Only after correct choices were the goal boxes shuffled among the three goal arms. Pretraining was given in blocks of 10 trials per day with $30 \mathrm{sec}$ intertrial intervals, the time it took to change the goal-box configuration. After a rat chose the correct box in 8 of 10 daily trials for 6 consecutive days ( $3 \mathrm{~d}$ per deprivation state), formal training began. Pretraining required $\sim 3$ weeks.

Training. Training in the contextual retrieval task was given in blocks of six trials per day with $10 \mathrm{~min}$ intertrial intervals. At the start of each trial, the rat was placed on the start arm and given access to the three goal arms. Only the goal box with the reward appropriate to the animal's current deprivation state was baited. After an incorrect choice, the rat was given $5-10$ sec to inspect the box and was then returned to its home cage for $\sim 30 \mathrm{sec}$. As during pretraining, incorrect trials were repeated with the same configuration of cue boxes until the correct choice was made. After a correct choice, the rat was allowed to consume the reward and was then returned to its home cage for $10 \mathrm{~min}$. Before the next trial, the boxes were moved to new locations determined by a pseudorandom sequence that ensured that each box occupied each goal arm twice daily. In this manner, each rat obtained food and water within an identical external environment and learned to select among equally reinforced approach responses on the basis of its internal motivational state.

Presurgery probe tests. To test whether rats were simply alternating their goal box selection from one day to the next, deprivation states were randomly repeated over $2 \mathrm{~d}$ throughout the later stages of training. To test whether rats were using reward odors to find food or water, random trials were run in which goal boxes were empty and reward was delivered only after the rat entered the correct box.

\section{Surgery}

After each rat reached an $80 \%$ correct choice criterion and maintained this level of performance for 6 consecutive days $(3 \mathrm{~d}$ per deprivation state), it was assigned to the control $(n=6)$, fornix $(n=7)$, or hippocampal $(n=5)$ lesion group. Four control animals were reassigned to the hippocampal lesion group after testing. Two rats were given hippocampal lesions after they had been performing at criterion levels for 2 months.

Surgical procedures used aseptic stereotaxic techniques. Each rat was anesthetized with isoflurane (Forane; Baxter, Deerfield, IL). Hippocampal animals were injected with $0.05 \mathrm{mg} / \mathrm{kg}$ atropine sulfate at the start of surgery. Bilateral radiofrequency lesions of the fornix were made using previously published methods (Ferbinteanu and Shapiro, 2003). Rats given fornix lesions were allowed $8 \mathrm{~d}$ of recovery before testing. Nonsurgical control animals were given equal time off the maze. Selective hippocampal lesions (Ammon's horn and dentate gyrus) were produced by 13 microinjections of ibotenate (IBO) $(10 \mu \mathrm{g} / \mu \mathrm{l}$; Sigma, St. Louis, MO) dissolved in PBS into each hippocampus (Jarrard, 1989). Control subjects had cannulas lowered into the cortex but were given no injections. At the completion of surgery, each rat was injected with normal saline ( 3 $\mathrm{ml}$, s.c.) and flunixin meglumine (1.5 mg/kg, i.m.). Control and hippocampal animals were given $10 \mathrm{~d}$ of recovery before testing.

\section{Postsurgical testing}

Testing procedures used after surgery were the same as in training, except that no correction procedure was used. Incorrect trials were not repeated.

\section{Postsurgical probe tests}

To determine whether rats discriminated internal states and were appropriately motivated during testing, each rat was assessed for item preference in a series of probe trials. A pair of small cups $(0.5 \mathrm{~cm}$ deep, $1.5 \mathrm{~cm}$ diameter) containing food and water was placed in the central platform of the maze. At the start of each trial, the deprived rat was placed on the start arm and given access to the central platform; the first consumed reward was then measured. This procedure was performed once for each rat in each deprivation condition (six trials per day).

\section{Histology}

After testing, each animal was deeply anesthetized with pentobarbital and perfused transcardially with PBS followed by $4 \%$ paraformaldehyde. Next, the brain was removed.

Fornix lesions. Brains were cryoprotected in $10 \%$ glycerol in $0.1 \mathrm{M}$ phosphate buffer (PB) for $1 \mathrm{~d}$ and then in $20 \%$ glycerol in $0.1 \mathrm{M}$ PB for an additional day. Brains were blocked, frozen, and sectioned at $50 \mu \mathrm{m}$ using a freezing-sliding microtome. Alternate sections were stained with formol-thionin to assess lesion size and placement. Remaining sections were stained for acetylcholinesterase (AChE) activity (Hedreen et al., 1985) to assess lesion completeness.

Hippocampal lesions. Brains were cryoprotected in $10 \%$ sucrose in 0.1 M PB for $1 \mathrm{~d}$ and then in $20 \%$ sucrose in $0.1 \mathrm{M} \mathrm{PB}$ for an additional day. Brains were blocked and sectioned at $40 \mu \mathrm{m}$ on a cryostat. Every fifth section was collected and stained with formol-thionin. Hippocampal lesion size and placement were assessed microscopically using StereoInvestigator software (MicroBrightField, Williston, VT). The amount of damage was expressed as the percentage of reduction in hippocampal tissue volume in lesioned animals compared with control animals. 
A

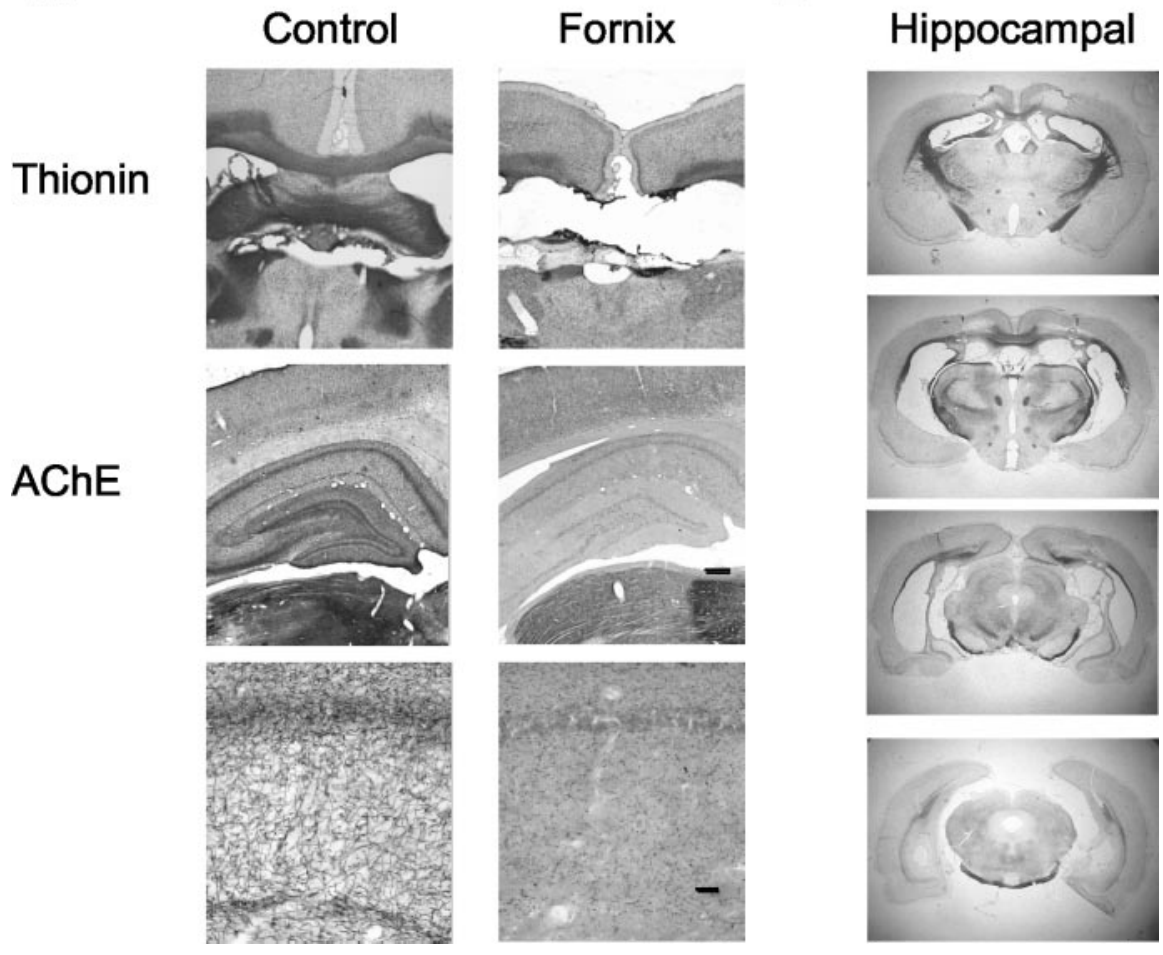

Figure 1. Photomicrographs of fornix and hippocampal lesions. A, Representative fornix lesion. Top, Sections stained with formol-thionin. Middle and bottom, Sections stained for AChE activity. High-power images of AChE show the stratum radiatum of CA1. Scale bars: middle, $250 \mu \mathrm{m}$; bottom, $25 \mu \mathrm{m}$. B, Sections from one representative ibotenate-lesioned rat showing the extent of hippocampal damage.

A

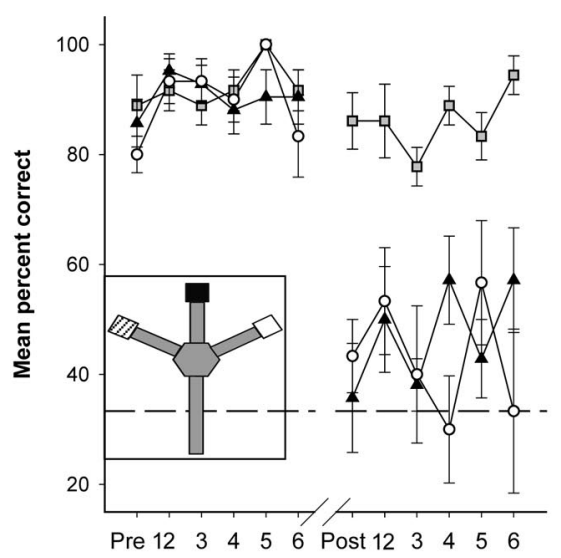

B

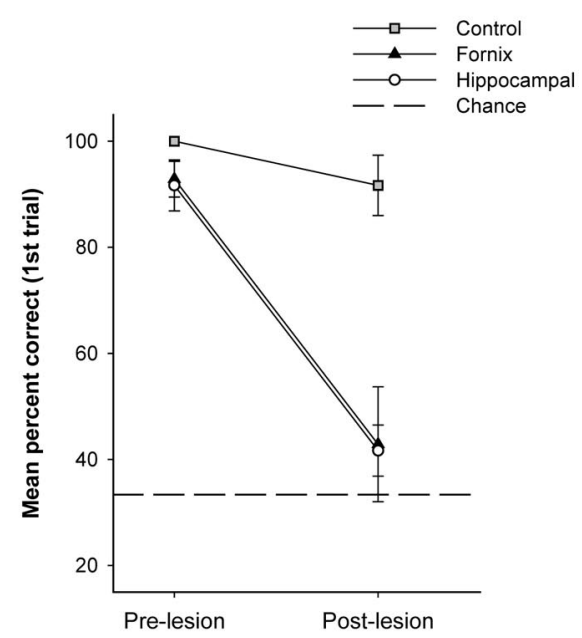

Figure 2. Performance scores in the contextual retrieval task. A, Mean percentage of correct scores from the last $6 \mathrm{~d}$ of training and the first $6 \mathrm{~d}$ of testing for control, fornix, and hippocampal groups. The inset shows a top-down schematic of the modified radial maze. Goal boxes were visually distinct, with one painted black, one painted white, and one painted white with a lit bulb illuminating its interior. $B$, Mean percentage of correct scores obtained from the first trials of the last $6 \mathrm{~d}$ of training and the first $6 \mathrm{~d}$ of testing for control, fornix, and hippocampal groups. Dashed lines indicate chance performance.

\section{Results}

\section{Lesion assessment}

The seven rats included in the fornix group had complete transection of the fornix and sustained minimal cortical damage at the point of entry of the electrodes. A representative fornix lesion is illustrated in Figure $1 \mathrm{~A}$. In all animals, the lesion dramatically reduced hippocampal AChE-positive fibers, indicating reduced cholinergic input to the hippocampus from the medial septum.

The five rats given bilateral ibotenic acid injections had extensive damage to the hippocampus and minimal damage to surrounding regions (Fig. $1 \mathrm{~B}$ ). Damage to the hippocampal subfields and dentate gyrus ranged from 84 to $96 \%$ of the total hippocampal volume averaged across controls. All rats had minor damage to the most anterior portions of the subicular complex and minimal damage to cortical regions immediately overlying the hippocampus. Two rats also had slight damage to the medial entorhinal cortex. Hippocampal tissue sparing was mainly in the ventral and most anterior dorsal areas of the hippocampus.

\section{Behavior}

Preoperative performance and probe tests Rats required an average of 72 training sessions from the start of habituation to the $80 \%$ criterion level of performance. Probe tests given before surgery revealed that rats were selecting responses on the basis of interoceptive cues rather than merely alternating their goal choices from one day to the next or using olfactory cues to locate rewards. When deprivation states were randomly repeated on 2 consecutive days, rats consistently selected the box that contained the deprived reward. Performance levels remained equally high when reward delivery was delayed until after the rat entered the correct box.

During the $6 \mathrm{~d}$ of training before surgery, all rats maintained similar levels of performance (Fig. 2A). To test whether rats were using working memory to return to a recently rewarded box, we also assessed the first response on each day (Fig. $2 B)$. Choice accuracy in the first response each day was nearly identical to the six trial means, demonstrating that rats were not using short-term working memory to guide behavior. Together, these tests verified that the rats were using internal contextual cues to guide goal-directed responses independent of the order of their presentation, reward-associated odor cues, or working memory.

Postoperative performance and probe tests Contextual memory retrieval required the hippocampal system. Both sham surgery and nonsurgical control groups maintained equally high levels of performance after surgery and were thus grouped together for statistical analysis. Control rats maintained criterion levels of performance throughout the $6 \mathrm{~d}$ of testing. In contrast, both lesion groups were severely impaired (Fig. 2A). A multivariate 
ANOVA confirmed that performance did not differ between control and lesion groups before surgery $\left(F_{(2,15)}=0.78 ; p>\right.$ $0.4)$ but did differ between the groups after surgery $\left(F_{(2,15)}=21.8 ; p<0.001\right)$. Fornix and hippocampal groups were similarly impaired (mean percentage correct over $6 \mathrm{~d}$ : fornix $=47.6$, hippocampus $=43.3$; Scheffe's post hoc test; $\mathrm{df}=15 ; p=0.81$ ), and each lesion group was significantly impaired relative to the control group (mean percentage correct over $6 \mathrm{~d}=86.1$; Scheffe's post hoc test; df $=15 ; p<$ $0.0001)$.

The deficit was not attributable to cue discrimination problems or impaired stimulus-reward associations. If the rats with lesions did not discriminate between the goal boxes or if they had lost the stimulus-reward associations that were acquired during training, then they should approach the three goal boxes with equal, random frequency. In fact, all three groups preferentially entered the wrong rewarded box rather than the nonrewarded box on error trials (Bonferroni corrected paired $t$ test, $5.4 \leq t \leq 7$; $p \leq$ 0.02 ) (Fig. 3A). However, the distribution of errors differed between the groups overall $\left(F_{(2,15)}=6.2 ; p<0.02\right)$. Control and fornix groups made a similar proportion of their errors to the wrong rewarded and nonrewarded boxes, and both differed from the hippocampal group, which entered the nonrewarded boxes somewhat more often (Scheffe's post hoc test; $\mathrm{df}=15 ; p \leq 0.05$ ). The only observable behavioral difference between the groups that may account for this dissimilarity was a more pronounced general hyperactivity in the rats with hippocampal lesions, perhaps as a consequence of cortical damage. Although the hippocampal rats did not adopt any consistent response biases or perseverative tendencies, they did move reliably faster on the maze than control or fornix rats. The rats with ibotenate lesions also appeared to swim faster than the other rats in a separate experiment in a water maze. Furthermore, although all rats learned to swim directly to a visible platform (swim distance, between-groups ANOVA; $F_{(2,15)}=1.5 ; p>0.25$ ), the rats with hippocampal or fornix lesions were impaired relative to controls when they had to find a hidden platform $\left(F_{(2,15)}=3.1 ; p<0.05\right.$; one-tailed). Both Fx and IBO groups were impaired relative to control (Fisher's least significant difference; $\mathrm{df}=15 ; p<0.05$; two-tailed). Thus, in contrast to control rats, those with lesions revealed the selective spatial learning deficit typically associated with hippocampal system lesions.

The lesions did not impair the rats' ability to discriminate between deprivation states. When deprived rats were given the choice between food and water on the maze (six trials per day; $1 \mathrm{~d}$ per deprivation state), every rat reliably first selected and consumed the item for which they were deprived, independent of lesion (Fig. 3B). The consistency of choice and consumption over the six probe trials further suggested that rats remained appropriately motivated throughout the day of testing. Thus, the contextual memory retrieval deficit cannot be attributed to a failure in motivation, stimulus-reward associative learning, or discrimination of either external or internal stimuli.
B
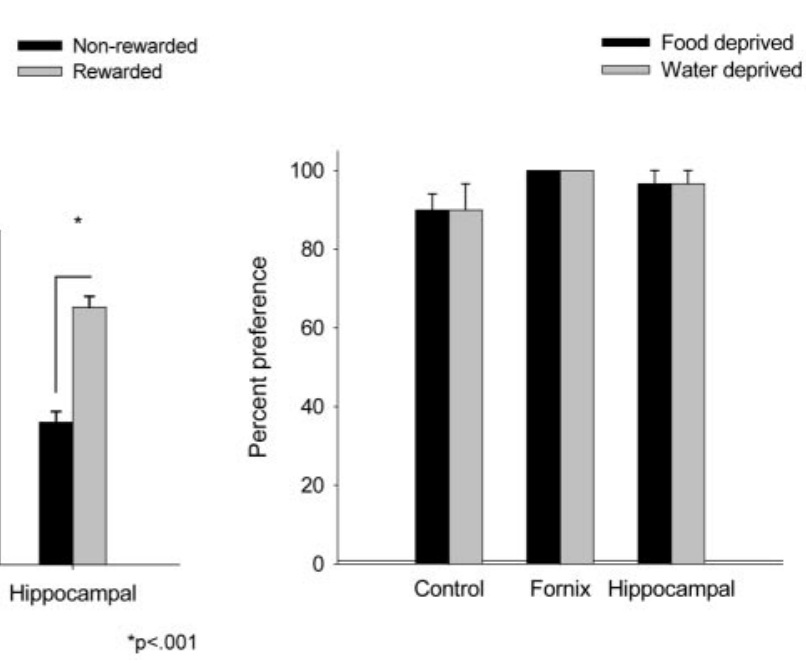

$* p<.001$

Figure 3. Error distribution and preference for the deprived item. $A$, Mean percentage of total errors committed and error type after fornix and hippocampal lesions. $B$, Mean percentage preference for deprived items. Percentage preference scores under each deprivation condition reflect the number of correct choices over six trials. Error bars in $A$ and $B$ show $1 \mathrm{SEM}$; L-shaped bars in $A$ indicate within-group statistical comparisons.

\section{Discussion}

\section{Summary}

Rats learned to use internal contextual cues derived from deprivation states to select among equally reinforced responses. Probe tests revealed that normal rats were using internal states to guide goal-directed responses rather than odor cues or working memory. Lesions of the fornix or the hippocampus severely impaired nonspatial contextual memory retrieval but preserved discrimination of interoceptive and exteroceptive cues as well as stimulus-reward associative learning. The hippocampus was therefore required for flexible memory retrieval based on internal context in a task that could not be solved by allocentric spatial strategies.

\section{Lesions of the hippocampal system}

Fornix and hippocampal lesions each impair hippocampal function but differ anatomically and functionally. Fornix transection disrupts subcortical-hippocampal connectivity while leaving hippocampal neurons and retrohippocampal connections intact. Excitotoxic lesions of the hippocampus selectively destroy neurons in the dentate gyrus and Ammon's horn while sparing fibers of passage. The two types of lesions also cause different types of unintended damage (McClelland et al., 1995; Alonso et al., 1996; Aggleton and Saunders, 1997). Although fornix and hippocampal lesions typically have similar effects on learning and memory, they can also differ (O'Keefe et al., 1975; O'Keefe and Nadel, 1978; McDonald et al., 1997). Ibotenate can demyelinate fibers close to the site of injection (Coffey et al., 1988). The ventral hippocampus and amygdala are in close anatomical proximity and are moderately interconnected (Pitkanen et al., 2000), and ibotenate may cause unintended demyelination of fibers en route to and from the amygdala. Here, hippocampal lesions increased general activity and slightly reduced a yet significant preference for the rewarded cues compared with fornix lesions. However, both fornix and hippocampal lesions similarly impaired contextual retrieval performance, demonstrating that the memory impairment was specifically caused by disruption of hippocampal function. Future studies are needed to determine the effects of 
selective lesions to either the ventral hippocampus or the amygdala on contextual memory retrieval.

Hippocampal function and representation of internal context In one of the first theories positing the existence of multiple memory systems in the mammalian brain, Hirsh (1974) proposed that interoceptive stimuli, among others, can provide contextual information by which memories can be retrieved and more importantly, that such contextual memory retrieval requires the hippocampus. However, previous studies required rats to use interoceptive retrieval cues (hunger or thirst) to discriminate spatial responses and did not determine whether the performance impairments were attributable to deficits in spatial information processing or contextual retrieval per se (Hirsh et al., 1978a,b, 1979). In the present study, neither allocentric nor egocentric spatial response strategies were necessary or sufficient for solving the task. The results show definitively that hippocampal lesions impair contextual memory retrieval even when both exteroceptive and interoceptive stimuli can be discriminated.

\section{Relational memory and contextual memory retrieval}

The observed deficits in contextual memory retrieval can be explained within the relational memory theory of hippocampal function. From this view, the hippocampus supports the rapid acquisition, storage, and retrieval of relational representations among all types of stimuli (Eichenbaum, 1999). Animal models of hippocampal function have typically focused on tasks in which performance depends on the ability to represent relationships among external stimuli. The present findings show that nonhuman animals can associate internal states with specific external stimuli and behaviors and that hippocampal damage impairs the selective and flexible retrieval of nonspatial information via these associations. Thus, in addition to spatial context that "contains and predicts" other stimuli (Nadel and Willner, 1980), the hippocampus encodes deprivation states and perhaps other internal contexts that select and predict the presence and significance of other stimuli.

\section{Encoding deprivation states}

How might deprivation state be represented in the brain so as to be associated with memory systems in general and the hippocampal system in particular? The hippocampus is both directly and indirectly connected with regions of the hypothalamus that are known to be crucial for regulating ingestive behaviors. The hippocampus receives dense innervation from neurons originating in the tuberomammillary nucleus and projections from orexin neurons originating in the lateral hypothalamic area, both of which have been implicated in feeding and drinking regulation (Kohler et al., 1985; Kunii et al., 1999; Sakurai, 1999; Brown et al., 2001). Hypothalamic connections with the hippocampus may convey functionally relevant information about internal state. The hypothalamic information may be encoded together with simultaneous and sequential inputs to construct detailed event memories. This content-addressable representation would then enable experiences of hunger and thirst to activate memories associated with food or water, including when, where, and which stimuli best predicted specific rewards. Once activated, hippocampal neural representations could serve to guide goaloriented behaviors. These ideas must be tested directly in the future.

\section{Contextual retrieval and learned associations}

Contextual retrieval can be viewed as a type of conditional association task that requires discrimination of one stimulus with a meaning that varies with another. Such tasks include negative patterning (Sutherland and Rudy, 1989; Alvarado and Rudy, 1995), biconditional discrimination learning (Whishaw and Tomie, 1991; Gallagher and Holland, 1992; Alvarado and Rudy, 1995; McDonald et al., 1997), and object-place associations (Gilbert and Kesner, 2002). Each of these conditional association tasks can, in principle, be solved by more than one strategy. For example, negative patterning tasks can be solved either by remembering the explicit relationships among the elements of a stimulus array (a relational strategy) or by responding to a compound stimulus that merges the elements of an array into a single configuration (a compound strategy) (Eichenbaum and Bunsey, 1995). Specific task parameters can influence the extent to which a given conditional association task is solved using relational or compound strategies. When task contingencies encourage rats to identify, compare, and respond differently to the same stimuli under different circumstances (e.g., the stimulus-response competition in negative patterning), then the rat is likely to encode the relationships between the stimuli in each situation. However, when task contingencies permit rats to solve tasks by combining stimuli into compounds and responding consistently to those compounds (e.g., biconditional discrimination learning), then the rat is likely to ignore relational complexity. Operationally, stimulus-response competition should encourage relational encoding, whereas stimulus-response consistency should encourage the formation of and response to compound stimuli (McDonald et al., 1997).

The present experiment required deprived rats to switch approach and avoidance responses in the presence of the same external stimuli depending on their current internal state and thus included stimulus-response competition. Reward was only available in a given box during trials associated with a specific deprivation state. Approaching the same box in the other deprivation state was not rewarded. The absence of consistent reward may have discouraged the formation of box-reward compound stimuli and encouraged the encoding of specific relationships among motivational cues, external stimuli, and specific rewards. From this view, motivational states served as conditional associative retrieval cues, specifying current box-reward relationships and thus the appropriate goal-directed behaviors. The present data are consistent with previous reports that rats with hippocampal damage are impaired in learning to use deprivation-state cues to predict shock in an otherwise ambiguous environment (Davidson and Jarrard, 1993; Hock and Bunsey, 1998). The cognitive demands of these tasks also required a conditional association whereby the rat's deprivation state specified whether or not the conditioning chamber would be paired with shock.

This view may also account for the different results obtained in another internally cued discrimination task in which rats were trained to displace one of two small objects for food or water (Deacon et al., 2001). Rats with hippocampal lesions learned successfully to displace the object covering the reward appropriate to the rats' deprivation state. The task differed in several ways from the current study. First, rats were trained postoperatively, which by definition encourages rats with hippocampal lesions to adopt a nonhippocampal strategy. Second, both goal objects were consistently associated with reward, which was always available independent of deprivation state (Deacon et al., 2001). The consistent availability of reward may have encouraged the formation of compound object-reward representations and eliminated stimulus-response competition, because all approaches to an object were rewarded (Deacon et al., 2001). Rats with hippocampal lesions could therefore solve the task by continuously updating the 
predicted value of each object based on their current deprivation state, a process shown to be mediated by the amygdala (Malkova et al., 1997; Baxter et al., 2000).

Although the observed impairment could derive, in principle, from impaired object-water-object-food associations or stimulus-response associations, these interpretations are unlikely. First, hippocampal damage does not impair learning about explicit, nonspatial stimulus-stimulus associations (Murray et al., 1993; Cho and Kesner, 1995; Bunsey and Eichenbaum, 1996). Second, well learned flavor-place associations or stimulus-response associations are retrieved normally by rats with fornix lesions or hippocampal inactivation, respectively (Murray and Wise, 1996; Brasted et al., 2003; Day et al., 2003). These experiments suggest that if training produced either simple stimulusstimulus or stimulus-response associations, then hippocampal lesions should spare memory performance. In the present experiment, the rats were trained extensively before surgery. In fact, some animals had performed the task at criterion levels for 2 months. In contrast to the experiments just described, lesions given after extensive training still impaired performance, suggesting that memory performance depended on processes other than simple box-reward or stimulus-response associative retrieval.

\section{"Episodic-like" memory and internal context}

Human episodic memory encodes the spatial, temporal, and contextual components of events so that detailed memories can be retrieved via a subset of event features (Tulving, 1972). Nonverbal animals may possess a form of episodic memory, defined operationally in tasks that require knowing what, where, and when (Clayton and Dickinson, 1998; Clayton et al., 2001; Morris, 2001). Contextual retrieval tasks provide an additional behavioral measure of episodic-like memory in nonhuman species. The present task required rats to distinguish between two distinct experiences within the same external environment. Performance was dependent on an associative reconstruction of the relationships among the elements that characterized each experience, namely the rats' internal state, external stimuli (e.g., the current goal), and specific rewards. The near-perfect performance of intact animals on the first trial of each daily session and the fact that both goal boxes were equally paired with reward and nonreward suggest that response selection was not based on familiarity with the current rewarded box or on box-reward associative strength (cf. Morris, 2001). This experiment adds to the growing repertoire of nonspatial, episodic-like memory tasks that require the hippocampus, and the results provide new insight into the scope of information encoded by the hippocampus in nonverbal animals.

\section{References}

Aggleton JP, Saunders RC (1997) The relationships between temporal lobe and diencephalic structures implicated in anterograde amnesia. Memory 5:49-71.

Alonso JR, U HS, Amaral DG (1996) Cholinergic innervation of the primate hippocampal formation. II. Effects of fimbria/fornix transection. J Comp Neurol 375:527-551.

Alvarado MC, Rudy JW (1995) A comparison of kainic acid plus colchicine and ibotenic acid-induced hippocampal formation damage on four configural tasks in rats. Behav Neurosci 109:1052-1062.

Baxter MG, Parker A, Lindner CC, Izquierdo AD, Murray EA (2000) Control of response selection by reinforcer value requires interaction of amygdala and orbital prefrontal cortex. J Neurosci 20:4311-4319.

Brasted PJ, Bussey TJ, Murray EA, Wise SP (2003) Role of the hippocampal system in associative learning beyond the spatial domain. Brain 126:1202-1223.
Brown RE, Stevens DR, Haas HL (2001) The physiology of brain histamine. Prog Neurobiol 63:637-672.

Bunsey M, Eichenbaum H (1996) Conservation of hippocampal memory function in rats and humans. Nature 379:255-257.

Cho YH, Kesner RP (1995) Relational object association learning in rats with hippocampal lesions. Behav Brain Res 67:91-98.

Clayton NS, Dickinson A (1998) Episodic-like memory during cache recovery by scrub jays. Nature 395:272-274.

Clayton NS, Griffiths DP, Emery NJ, Dickinson A (2001) Elements of episodic-like memory in animals. Philos Trans R Soc Lond B Biol Sci 356:1483-1491.

Coffey PJ, Perry VH, Allen Y, Sinden J, Rawlins JN (1988) Ibotenic acid induced demyelination in the central nervous system: a consequence of a local inflammatory response. Neurosci Lett 84:178-184.

Cohen NJ, Eichenbaum H (1993) Memory, amnesia, and the hippocampal system. Cambridge, MA: MIT.

Davidson TL, Jarrard LE (1993) A role for hippocampus in the utilization of hunger signals. Behav Neural Biol 59:167-171.

Day M, Langston R, Morris RG (2003) Glutamate-receptor-mediated encoding and retrieval of paired-associate learning. Nature 424:205-209.

Deacon RM, Bannerman DM, Rawlins NP (2001) Conditional discriminations based on external and internal cues in rats with cytotoxic hippocampal lesions. Behav Neurosci 115:43-57.

Eichenbaum H (1999) The hippocampus and mechanisms of declarative memory. Behav Brain Res 103:123-133.

Eichenbaum HB, Bunsey M (1995) On the binding of associations in memory: clues from studies on the role of the hippocampal region in pairedassociate learning. Curr Dir Psychol Sci 4:19-23.

Ferbinteanu J, Shapiro ML (2003) Prospective and retrospective memory coding in the hippocampus. Neuron 40:1227-1239.

Gallagher M, Holland PC (1992) Preserved configural learning and spatial learning impairment in rats with hippocampal damage. Hippocampus 2:81-88.

Gilbert PE, Kesner RP (2002) Role of the rodent hippocampus in pairedassociate learning involving associations between a stimulus and a spatial location. Behav Neurosci 116:63-71.

Hedreen JC, Bacon SJ, Price DL (1985) A modified histochemical technique to visualize acetylcholinesterase-containing axons. J Histochem Cytochem 33:134-140.

Hirsh R (1974) The hippocampus and contextual retrieval of information from memory: a theory. Behav Biol 12:421-444.

Hirsh R, Leber B, Gillman R (1978a) Fornix fibers and motivational states as controllers of behavior: a study stimulated by the contextual retrieval theory. Behav Biol 22:463-475.

Hirsh R, Holt L, Mosseri A (1978b) Hippocampal mossy fibers, motivational state, and contextual retrieval. Exp Neurol 62:68-79.

Hirsh R, Davis RE, Holt L (1979) Fornix-thalamus fibers, motivational states, and contextual retrieval. Exp Neurol 65:373-390.

Hock BJ, Bunsey MD (1998) Differential effects of dorsal and ventral hippocampal lesions. J Neurosci 18:7027-7032.

Hsiao S, Isaacson RL (1971) Learning of food and water positions by hippocampus damaged rats. Physiol Behav 6:81-83.

Jarrard LE (1989) On the use of ibotenic acid to lesion selectively different components of the hippocampal formation. J Neurosci Methods 29:251-259.

Kohler C, Swanson LW, Haglund L, Wu JY (1985) The cytoarchitecture, histochemistry and projections of the tuberomammillary nucleus in the rat. Neuroscience 16:85-110.

Kunii K, Yamanaka A, Nambu T, Matsuzaki I, Goto K, Sakurai T (1999) Orexins/hypocretins regulate drinking behaviour. Brain Res 842:256-261.

Malkova L, Gaffan D, Murray EA (1997) Excitotoxic lesions of the amygdala fail to produce impairment in visual learning for auditory secondary reinforcement but interfere with reinforcer devaluation effects in rhesus monkeys. J Neurosci 17:6011-6020.

McClelland JL, McNaughton BL, O’Reilly RC (1995) Why there are complementary learning systems in the hippocampus and neocortex: insights from the successes and failures of connectionist models of learning and memory. Psychol Rev 102:419-457.

McDonald RJ, Murphy RA, Guarraci FA, Gortler JR, White NM, Baker AG (1997) Systematic comparison of the effects of hippocampal and fornix- 
fimbria lesions on acquisition of three configural discriminations. Hippocampus 7:371-388.

Morris RG (2001) Episodic-like memory in animals: psychological criteria, neural mechanisms and the value of episodic-like tasks to investigate animal models of neurodegenerative disease. Philos Trans R Soc Lond B Biol Sci 356:1453-1465.

Murray EA, Wise SP (1996) Role of the hippocampus plus subjacent cortex but not amygdala in visuomotor conditional learning in rhesus monkeys. Behav Neurosci 110:1261-1270.

Murray EA, Gaffan D, Mishkin M (1993) Neural substrates of visual stimulus-stimulus association in rhesus monkeys. J Neurosci 13:4549-4561.

Nadel L, Willner J (1980) Context and conditioning: a place for space. J Comp Physiol Psychol 8:218-228.

O'Keefe J, Nadel L (1978) The hippocampus as a cognitive map. Oxford: Oxford UP.

O’Keefe, Nadel L, Keightely S, Kill D (1975) Fornix lesions selectively abolish place learning in the rat. Exp Neurol 48:152-166.

Pitkanen A, Pikkarainen M, Nurminen N, Ylinen A (2000) Reciprocal connec- tions between the amygdala and the hippocampal formation, perirhinal cortex, and postrhinal cortex in rat. A review. Ann NY Acad Sci 911:369-391.

Sakurai T (1999) Orexins and orexin receptors: implication in feeding behavior. Regul Pept 85:25-30.

Scoville WB, Milner B (1957) Loss of recent memory after bilateral hippocampal lesions. J Neurol Neurosurg Psychiatry 20:11-21.

Sutherland RJ, Rudy JW (1989) Configural association theory: the role of the hippocampal formation in learning, memory and amnesia. Psychobiology 17:129-144.

Tulving E (1972) Episodic and semantic memory. In: Organization of memory (Tulving E, Donaldson W, eds), pp 382-403. New York: Academic.

Vargha-Khadem F, Gadian DG, Watkins KE, Connelly A, Van Paesschen W, Mishkin M (1997) Differential effects of early hippocampal pathology on episodic and semantic memory. Science 277:376-380.

Whishaw IQ, Tomie JA (1991) Acquisition and retention by hippocampal rats of simple, conditional, and configural tasks using tactile and olfactory cues: implications for hippocampal function. Behav Neurosci 105:787-797. 